\title{
Ex vivo pyelotomy, nephroscopy and holmium laser lithotripsy of a staghorn stone in a donor kidney prior to renal transplant
}

\author{
Dariusz Janczak ${ }^{1,2}$, Barbara Bolanowska ${ }^{1}$, Paweł Jankowski ${ }^{1}$, Tadeusz Dorobisz ${ }^{1}$, Karolina Dorobisz ${ }^{3}$, \\ Mariusz Chabowski ${ }^{1}$, Dawid Janczak ${ }^{4}$, Tomasz Szydelko ${ }^{4,5}$ \\ ${ }^{1}$ Department of Surgery, Fourth Military Teaching Hospital, Wroclaw, Poland \\ ${ }^{2}$ Department of Clinical Proceedings, Faculty of Health Science, Wroclaw Medical University, Wroclaw, Poland \\ ${ }^{3}$ Department of Otolaryngology, Head and Neck Surgery, Wroclaw Medical University, Wroclaw, Poland \\ ${ }^{4}$ Department of Palliative Care Nursing, Wroclaw Medical University, Wroclaw, Poland \\ ${ }^{5}$ Clinical Department of Urology, $4^{\text {th }}$ Clinical Military Hospital, Wroclaw, Poland
}

Videosurgery Miniinv 2015; 10 (2): 286-289

DOI: $10.5114 /$ wiitm.2015.52556

\begin{abstract}
This case report presents the diagnostic and treatment procedures of stone removal from the kidney of a 67-year-old donor, the transplantation of the kidney to a 65-year-old recipient, and the postoperative course until the end of hospitalization. Computed tomography performed before collecting the organ showed a staghorn stone in the renal pelvis and lower calyces in the right donor kidney. The stones were removed ex-vivo using a rigid ureteroscope and a holmium laser prior to transplantation. Then the organ was transplanted to the left iliac fossa of a 65-year-old man with end-stage renal failure. The authors think there is a possibility of increasing the kidney pool, by using organs containing large calculi. In such cases stones should be removed before the operation and the patient should be monitored regularly, especially in the first months after the transplant.
\end{abstract}

Key words: renal transplant, nephrolithiasis, laser lithotripsy.

\section{Introduction}

Nephrolithiasis affects approximately $1.5 \%$ of the European population [1]. Stones located in the renal collecting system are not a common clinical problem in transplanted organs. Their presence can be diagnosed prior to transplantation, in the early postoperative period or in a functioning transplant. In the latter case stones are formed de novo. Nephrolithiasis after transplantation is a very rare clinical problem: it occurs in $0.17 \%$ to $1.8 \%$ of patients. It may impair renal function and cause urinary tract infections. Nephrolithiasis in kidneys scheduled for transplantation or in the active transplant always constitutes a significant clinical challenge. So far, case reports concerning small non-obstructive calculi have been published. To the best of our knowledge, the following study is the first to present staghorn stone removal from a donor kidney before transplant.

\section{Case report}

The following case report presents the clinical identification, treatment and postoperative management of the right kidney with staghorn nephrolithiasis taken from a 67-year-old donor, which was transplanted to a 65-year-old recipient. The donor was a man with hypertension, obesity and chronic obstructive pulmonary disease. The cause of death was ischemic brain damage after cardiac arrest.

US performed in the potential donor showed a staghorn stone in the right renal pelvis and lower 
calyx. Urine culture was sterile. Computed tomography (CT) performed after the procurement showed a staghorn stone in the right renal pelvis, with dimensions of $27 \times 18 \mathrm{~mm}$ and two other stones in the lower calyx with dimensions of $14 \times 9 \mathrm{~mm}$ and $11 \times 9 \mathrm{~mm}$ (Photo 1). The etiology of nephrolithiasis was not analyzed. The donor kidney was biopsied to assess the number of fibrous glomeruli and to evaluate the condition of the renal interstitium. Histology revealed no abnormalities.

Following the procurement, the kidney was placed in sterile, iced preservation solution. As the stone in the renal pelvis was large, the decision was made to perform U-shaped pyelotomy to remove it. Then a $6.5 \mathrm{Fr}$ rigid ureteroscope was introduced to the lower calyx through the incision of the renal pelvis and two stones were localized under direct vision. The intra-operative view was compared to preoperatively performed CT scans to precisely identify the stones. The calculi were fragmented with a holmium laser and removed using an endoscopic basket. During the whole procedure the kidney was kept cold in an iced saline bath. Once nephroscopy was completed, the renal pelvis and the calyces were flushed, a "double J" stent was left indwelling and the pyelotomy was closed with a 4-0 monofilament running suture It took $35 \mathrm{~min}$ to remove the stone from the transplanted kidney. Cold ischemia time (CIT) was $23 \mathrm{~h}$ and $30 \mathrm{~min}$, including CT of the kidney and histology of the renal core.

The kidney was transplanted to the 65-year-old man, who had been dialyzed for 9 months because of end-stage renal disease caused by hypertensive nephropathy. Additionally, the recipient was diagnosed with ischemic heart disease and prostatic hypertrophy. The patient had had two myocardial infarctions treated with percutaneous coronary intervention $(\mathrm{PCl})$ with stent placement. Cardiology consultation during transplant qualification revealed myocardial damage caused by hypertension without contraindications for transplantation.

The donor right kidney, after ex vivo stone removal, was transplanted into the left iliac fossa of the recipient. Perfusion fluid culture as well as swabs from the renal pelvis available $72 \mathrm{~h}$ after transplantation were sterile. The immunosuppression regimen consisted of antibody induction therapy, basiliximab, tacrolimus, mofetil mycophenolate and glucocorticoids. The clinical condition of the patient after the operation was good, but persistent ol- iguria, causing retention of nitrogenous metabolites, required several cycles of hemodialysis. Diuresis in the first $24 \mathrm{~h}$ after surgery was $250 \mathrm{ml}$. Oliguria lasted for 7 days after surgery. The transplanted kidney might have had delayed function because of a long cold ischemia time. From the seventh day on, diuresis gradually improved. Hematuria associated with anemia was an additional problem, but it resolved after 2 weeks of conservative treatment. Because of elevated serum $C$-reactive protein reaction (CRP) levels, empiric antibiotic therapy was administered, even though urine culture was sterile. After 7-day treatment, CRP returned to normal values.

The Foley catheter was removed on the $17^{\text {th }}$ day after transplantation. Ultrasound of the transplanted kidney was carried out after surgery. No evidence of stones, dilatation of the pelvicalyceal system or pathologic fluid reservoirs in the area of the graft were found. Perfusion flow in the transplant vessels was found normal. If US showed no abnormalities, CT was not performed.

On the $12^{\text {th }}$ postoperative day, arrhythmias occurred in the form of atrial fibrillation, with an in-

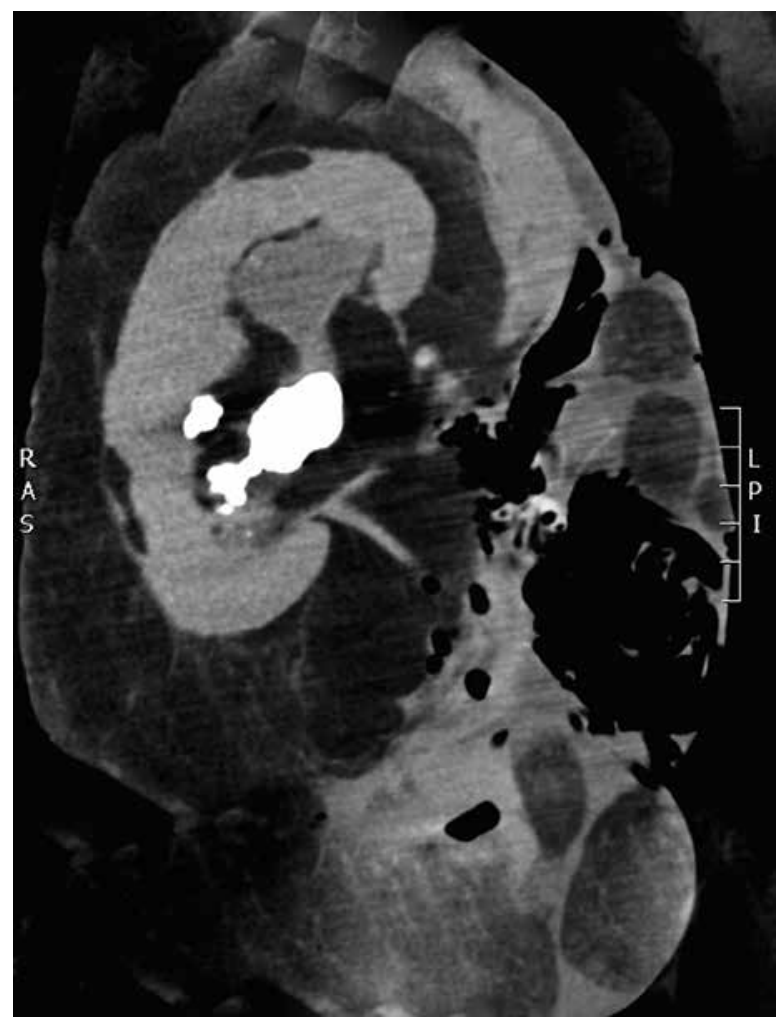

Photo 1. Computed tomography of donor kidney closed in a plastic bag with iced preservation solution 
crease in myocardial necrosis markers. The patient was treated in the cardiac intensive care unit with a final diagnosis of non ST-segment elevation myocardial infarction (NSTEMI). Assessing the whole clinical state, invasive treatment was abandoned. Instead of it, treatment with two antiplatelet drugs was implemented, without any bleeding complications. During hospitalization, a urinary tract infection was diagnosed and treated, with a good outcome. Ultrasound examination of the transplanted kidney did not reveal hydronephrosis or residual nephrolithiasis. On the $28^{\text {th }}$ day after the operation the patient was discharged from hospital in a good general condition. His serum creatinine was $2 \mathrm{mg} / \mathrm{dl}$ (eGFR $33 \mathrm{ml} / \mathrm{min}$ ). There were no symptoms of kidney disorders.

\section{Discussion}

Nephrolithiasis is a relative contraindication to kidney transplantation, both from a deceased as well as a living donor. It may cause an obstruction of the urinary tract and recurrent infections leading to function loss of the transplant [2]. Cases when stones are formed de novo or transferred with the donor organs have been reported. It should be emphasized that there are no diagnostic and therapeutic standards which pertain to detection and treatment of nephrolithiasis in kidney donors [3]. In some transplantology wards, computed tomography of the whole body is performed to reveal hidden pathologies [4]. The findings of the Mayo Clinic study indicate that $11 \%$ of potential living kidney donors who underwent $\mathrm{CT}$ had radiographic stones [5]. However, the most common imaging technique is ultrasonography of the abdomen. This diagnostic method should detect nephrolithiasis if present. The method of treating urolithiasis depends on the size and location of calculi. A therapeutic procedure may be performed ex vivo or in vivo in the recipient a few months after the organ transplantation. The recommended method of stone removal is endoscopic treatment. Only small stones can be removed with extracorporeal shock wave lithotripsy [4]. Removing calculi after transplantation is associated with numerous potential complications due to immunosuppressive therapy. Because of denervation, patients do not have symptoms of renal colic, which may cause diagnostic difficulties. Patients with nephrolithiasis in the transplanted kidney can easily develop chronic rejection, ureteral obstruction or recurrent urinary tract infections [6-8].
Recent advances in endourology have contributed to successful management of uncommon cases of urinary tract calculi [9]. Ex vivo ureteroscopy with holmium laser lithotripsy (exURS) of small, non-obstructive stones has been reported to be an effective procedure [10]. Lithotripsy is becoming a part of transplantation. The combined surgery reduces the risk of infections in the transplanted kidney and complications of multiple anesthesia. It also expands the donor pool, as marginal kidneys can be selected for transplant. Complications of ex vivo nephrolithotripsy such as damage of the ureter or renal pelvis have rarely been reported [2]. No major urinary tract complications were observed in the reported case in the post-operative period, either. Yet, it is noteworthy that exURS was performed for staghorn nephrolithiasis, not for a small, non-obstructive stone. Serum creatinine at discharge was $2 \mathrm{mg} / \mathrm{dl}$, the result being similar to the reported ones [2].

We did not find the exact reason for hematuria, but some potential reasons may be taken into consideration - benign prostate hypertrophy in a patient with a Foley catheter, pyelotomy performed during stone removal or ureterovesical anastomosis. The decision was made to treat the patient conservatively, and hematuria subsided after 2 weeks.

\section{Conclusions}

Ex-vivo endoscopic laser lithotripsy of staghorn renal stones in a donor kidney before transplantation is a safe and technically feasible procedure which may expand the kidney donor pool. In the authors' opinion, in cases of staghorn lithiasis, pyelotomy should be considered, as endoscopy alone might be difficult and time-consuming.

\section{Conflict of interest}

The authors declare no conflict of interest.

\section{References}

1. Kupajski M, Tkocz M, Ziaja D. Modern management of stone disease in patients with a solitary kidney. Videosurgery Miniinv 2012; 7: 1-7.

2. Rashid MG, Konnak JW, Stuart Wolf J Jr, et al. Ex vivo ureteroscopic treatment of calculi in donor kidneys at renal transplantation. J Urol 2004; 171: 58-60.

3. Klingler HC, Kramer G, Lodde M, Marberger M. Urolithiasis in allograft kidneys. Urology 2002; 59: 344-8.

4. Wong KA, Olsburgh J. Management of stones in renal transplant. Curr Opin Urol 2013; 23: 175-9. 
5. Lorenz EC, Lieske JC, Vrtiska TJ, et al. Clinical characteristics of potential kidney donors with asymptomatic kidney stones. Nephrol Dial Transplant 2011; 26: 2695-700.

6. Lu HF, Shekarriz B, Stoller ML. Donor-gifted allograft urolithiasis: early percutaneous management. Urology 2002; 59: 25-7.

7. Wheatley M, Ohl DA, Sonda LP, et al. Treatment of renal transplant stones by extracorporeal shock-wave litotripsy in the prone position. Urology 1991; 37: 57-60.

8. Bhadauria RP, Ahlawat R, Kumar RV, et al. Donor-gifted allograft lithiasis: extracorporeal shockwave litotripsy with over table module using the Lithostar Plus. Urol Int 1995; 55: 51-5.

9. Kupajski M, Tkocz MT. Use of endoscopic lithotripsy technique in the treatment of intestinal neobladder lithiasis performed by means of the VIP method. Videosurgery Miniinv 2014; 9: 93-5.

10. Schade GR, Wolf JS, Faerber GJ. Ex-vivo ureteroscopy at the time of live donor nefrectomy. J Endourol 2011; 25: 1405-9.

Received: 4.02.2015, accepted: 13.05.2015 